\title{
纳米 $\alpha-\mathrm{Fe}_{2} \mathrm{O}_{3} /(\mathrm{IPDI}-\mathrm{HTPB})$ 复合粒子的制备及其催化性能研究
}

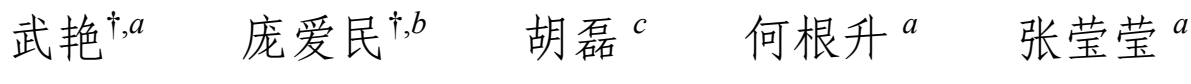 \\ 张利雄 ${ }^{c}$ 李明海 $*, a$ 马振叶 $*, a$ \\ ( ${ }^{a}$ 南京师范大学化学与材料科学学院 南京 210097) \\ ( ${ }^{b}$ 湖北航天化学技术研究所 航天化学动力技术重点实验室 襄阳 441003) \\ (c 南京工业大学材料化学工程国家重点实验室＼cjkstart南京 210009)
}

\begin{abstract}
摘要 为改善纳米 $\alpha-\mathrm{Fe}_{2} \mathrm{O}_{3}$ 在复合推进剂中的分散性, 提高其催化性能, 选择推进剂的配方组分固化剂异氟尔酮二异氧 酸酯(IPDI)为嫁接桥梁, 将端差基聚丁二烯(HTPB)接枝在纳米 $\alpha-\mathrm{Fe}_{2} \mathrm{O}_{3}$ 粒子的表面, 制得纳米 $\alpha-\mathrm{Fe}_{2} \mathrm{O}_{3} /$ (IPDI-HTPB)复合 粒子. 首先研究了 IPDI 与 HTPB 反应动力学, 优化制备条件; 然后采用 X 射线衍射仪 (XRD)、透射电子显微镜(TEM)、 傅里叶变换红外光谱仪(FTIR)和热重分析(TGA)等手段对复合粒子的结构进行表征, 采用 DTA 对比了复合前后纳米 $\alpha-\mathrm{Fe}_{2} \mathrm{O}_{3}$ 粒子对高氯酸铵(AP)热分解的催化性能的影响. 结果表明, 依靠 IPDI 的偶联作用, 可将 HTPB 接枝在纳米 $\alpha-\mathrm{Fe}_{2} \mathrm{O}_{3}$ 的表面, 包覆层厚度约为 $5 \mathrm{~nm}$, 改性后的纳米 $\alpha-\mathrm{Fe}_{2} \mathrm{O}_{3} /$ (IPDI-HTPB)复合粒子的分散性大幅提高, 纳米 $\alpha-\mathrm{Fe}_{2} \mathrm{O}_{3} /$ (IPDI-HTPB) 复合粒子对 AP 的热分解的催化性能也明显优于纯纳米 $\alpha-\mathrm{Fe}_{2} \mathrm{O}_{3}$ 粒子.
\end{abstract}

关键词 $\alpha-\mathrm{Fe}_{2} \mathrm{O}_{3}$; 端羟基聚丁二烯( $\left.\mathrm{HTPB}\right) ;$ 纳米复合粒子; 催化性能

\section{Preparation of $\alpha-\mathrm{Fe}_{2} \mathrm{O}_{3}$ /(IPDI-HTPB) Composite Nanoparticles and Their Catalytic Performance}

\author{
$\mathrm{Wu}, \mathrm{Yan}^{\dagger, a}$ \\ Pang, Aimin ${ }^{\dagger, b}$ \\ Zhang, Lixiong ${ }^{c}$ \\ Hu, Lei ${ }^{c} \quad H e$, Gensheng $^{a}$ \\ $\mathrm{Li}$, Minghai*,a \\ Ma, Zhenye ${ }^{*, a}$ \\ ( ${ }^{a}$ College of Chemical and Material Science, Nanjing Normal University, Nanjing 210097) \\ ( ${ }^{b}$ Science and Technology on Aerospace Chemical Power Laboratory, Institute of Aerospace Chemotechnology, \\ Xiangyang 441003)
}

( ${ }^{c}$ State Key Laboratory of Material-Oriented Chemical Engineering, Nanjing University of Technology, Nanjing 210009)

\begin{abstract}
In ammonium perchlorate (AP) based composite propellants, $\alpha-\mathrm{Fe}_{2} \mathrm{O}_{3}$ nanoparticles and hydroxyl terminated polybutadiene (HTPB) are commonly used as catalyst and binder respectively. Their properties and dispersion significantly affect the combustion performance of the composite propellants. However, $\alpha-\mathrm{Fe}_{2} \mathrm{O}_{3}$ nanoparticles are hard to disperse uniformly in the binder HTPB owing to the high viscosity, which will decrease their catalytic activity. Directly composite processing of $\alpha-\mathrm{Fe}_{2} \mathrm{O}_{3}$ nanoparticles with other main components of composite propellants may be an effective strategy to prevent from aggregation without introducing other components to the solid propellant at the same time. In this paper, the $\alpha-\mathrm{Fe}_{2} \mathrm{O}_{3} /$ (IPDI-HTPB) composite nanoparticles were prepared by choosing curing agent isophorone diisocyanate (IPDI) as grafting bridge. The typical procedure was as follows: (1) The first step was to synthesize IPDI-HTPB. In order to adjudged the reaction terminal point of IPDI and HTPB, the reaction kinetics of HTPB and IPDI were first researched and the proper reaction conditions were chosen as follows: molar ratio of HTPB and IPDI is $1: 1$, reaction temperature is under $80{ }^{\circ} \mathrm{C}$ and reaction time is $2 \mathrm{~h}$. (2) The second step was to synthesize $\alpha-\mathrm{Fe}_{2} \mathrm{O}_{3} /($ IPDI-HTPB) composite nanoparticles. Firstly, the stoichiometric $\alpha-\mathrm{Fe}_{2} \mathrm{O}_{3}$ nanoparticles were dispersed in toluene under ultrasound for $10 \mathrm{~min}$. Secondly, the mixture were added in the above IPDI-HTPB solution and the reaction kept for $4 \mathrm{~h}$. Thirdly, the $\alpha-\mathrm{Fe}_{2} \mathrm{O}_{3}$ (IPDI-HTPB) composite nanoparticles were centrifuged and washed with toluene and ether for several times. Finally, $\alpha-\mathrm{Fe}_{2} \mathrm{O}_{3} /(\mathrm{IPDI}-\mathrm{HTPB})$ composite nanoparticles were dried in the oven at $80{ }^{\circ} \mathrm{C}$ for $12 \mathrm{~h}$. (3) The structure of $\alpha$ - $\mathrm{Fe}_{2} \mathrm{O}_{3} /($ IPDI-HTPB) composite nanoparticles were characterized by X-ray diffractometer (XRD), transmittance electron microscopy (TEM), Fourier transform infrared spectrometer (FTIR) and thermogravimetric analysis (TGA). It was observed that HTPB could be chemically coated on the surface of the $\alpha-\mathrm{Fe}_{2} \mathrm{O}_{3}$ nanoparticles by the grafting activity of IPDI. The depth of the IPDI-HTPB was nearly $5 \mathrm{~nm}$. The $\alpha-\mathrm{Fe}_{2} \mathrm{O}_{3}$ /(IPDI-HTPB) composite nanoparticles showed hydrophobicity after the composite process. Compare with the pure $\alpha-\mathrm{Fe}_{2} \mathrm{O}_{3}$ nanoparticles, $\alpha-\mathrm{Fe}_{2} \mathrm{O}_{3}$ nanoparticles in $\alpha-\mathrm{Fe}_{2} \mathrm{O}_{3} /$ IPDI-HTPB composite nanoparticles showed better catalytic activity on the thermal decomposition of AP.
\end{abstract}

*E-mail:1mh741998@163.com; 07197@njnu.edu.cn

$\uparrow$ These authors contributed equally to this work.

Received December 9, 2019; published March 11, 2020.

Project supported by the Science and Technology on Aerospace Chemical Power Laboratory (No. STACPL120181B02-2).

项目受航天化学动力技术重点实验室开放基金(No. STACPL120181B02-2)资助. 
Keywords $\alpha-\mathrm{Fe}_{2} \mathrm{O}_{3}$ nanoparticles; hydroxyl terminated polybutadiene (HTPB); composite nanoparticles; catalytic activity

\section{1 引言}

三氧化二铁 $\left(\alpha-\mathrm{Fe}_{2} \mathrm{O}_{3}\right)$ 是一种重要的过渡金属氧化 物，广泛应用于催化、颜料、生物医药以及复合推进剂 等领域 ${ }^{[1 \sim 9]}$. 在复合推进剂领域, $\alpha-\mathrm{Fe}_{2} \mathrm{O}_{3}$ 是常用的催化 剂, 其纳米化被认为是一种提高推进剂燃烧性能的重要 方法. 研究表明 ${ }^{[10 \sim 12]}$, 处于纳米尺度的 $\alpha-\mathrm{Fe}_{2} \mathrm{O}_{3}$ 粒径小、 比表面大、表面能高, 表现出很高的催化活性. 但推进 剂常用的粘结剂如端羟基聚丁二烯(HTPB)粘性很大, 纳米催化剂在其中很难均匀分散, 会大大限制纳米催化 剂性能的提高, 给其实际应用带来困难.

为了解决纳米 $\alpha-\mathrm{Fe}_{2} \mathrm{O}_{3}$ 粒子的分散性, 国内外研究 者主要是对其进行表面改性 ${ }^{[10,13 \sim 15]}$. 但表面改性所用到 的改性剂往往不是推进剂的配方组分, 其引入会对推进 剂的性能产生潜在的不利影响. 将纳米催化剂与推进剂 固有组分复合是一种解决纳米催化剂团聚的有效方 法 ${ }^{[16 ~ 20]}$. 若将纳米 $\alpha-\mathrm{Fe}_{2} \mathrm{O}_{3}$ 粒子和 HTPB 进行复合, 制 备纳米复合粒子, 则可以在不引入推进剂非配方组分的 前提下, 有效提高纳米 $\alpha-\mathrm{Fe}_{2} \mathrm{O}_{3}$ 粒子在粘结剂中的分散 性和催化性能, 从而提高推进剂的燃烧性能. 本课题 组 ${ }^{[20]}$ 曾采用共混法制得 $\alpha-\mathrm{Fe}_{2} \mathrm{O}_{3} / \mathrm{HTPB}$ 复合粒子, 其中 纳米 $\alpha-\mathrm{Fe}_{2} \mathrm{O}_{3}$ 粒子和 HTPB 是靠物理作用结合的, 但在 后续的材料加工中, 容易出现表面 HTPB 脱落的现象, 且复合粒子结构的均一性以及稳定性都需要进一步提 高.

鉴于纳米 $\alpha-\mathrm{Fe}_{2} \mathrm{O}_{3}$ 粒子与 HTPB 直接反应比较困难, 本文尝试选取推进剂常用的固化剂异佛尔酮二异氧酸 酯(IPDI) 作为嫁接桥梁, 应用表面接枝法制备纳米
$\alpha-\mathrm{Fe}_{2} \mathrm{O}_{3} /(\mathrm{IPDI}-\mathrm{HTPB})$ 复合粒子, 示意图见图 1 所示. 采 用 $\mathrm{NCO}$ 浓度分析法对 HTPB 与 IPDI 的反应动力学进行 研究, 首先制备出 IPDI-HTPB, 然后再将纳米 $\alpha-\mathrm{Fe}_{2} \mathrm{O}_{3}$ 粒子与 IPDI-HTPB 进行反应来制备纳米 $\alpha-\mathrm{Fe}_{2} \mathrm{O}_{3} /$ (IPDI$\mathrm{HTPB}$ )复合粒子. 用 $\mathrm{X}$ 射线衍射仪(XRD)、透射电子显 微镜(TEM)、傅里叶变换红外光谱仪(FTIR)和热重分析差热分析法(TG-DTA)等手段对粒子形态与结构进行了 表征. 结果表明: 通过 IPDI 的偶联作用, HTPB 可成功 地嫁接在纳米 $\alpha-\mathrm{Fe}_{2} \mathrm{O}_{3}$ 表面; 表面包覆层厚度约为 $5 \mathrm{~nm}$; 改性后的 $\alpha-\mathrm{Fe}_{2} \mathrm{O}_{3}$ 复合粒子分散性得到大幅提高; 相比 于纯纳米 $\alpha-\mathrm{Fe}_{2} \mathrm{O}_{3}$ 粒子, 纳米 $\alpha-\mathrm{Fe}_{2} \mathrm{O}_{3}$ (IPDI-HTPB)复合 粒子对高氯酸铵(AP)的热分解具有更好的催化作用.

\section{2 实验结果与讨论}

\subsection{IPDI 与 HTPB 反应动力学研究}

在制备 IPDI-HTPB 大分子时如何确定 IPDI 反应完 全是必须研究的难题, 当溶液中存在未反应的 IPDI 时, 它会同纳米 $\alpha-\mathrm{Fe}_{2} \mathrm{O}_{3}$ 表面的 $\mathrm{OH}$ 反应而占据活性位，这 样便会影响 IPDI-HTPB 大分子与纳米 $\alpha-\mathrm{Fe}_{2} \mathrm{O}_{3}$ 的接枝. 因而, 我们对 IPDI 与 HTPB 反应的动力学进行了研究, 考察了两者反应的终点条件和反应的活化能.

IPDI 分子中存在 2 个 $\mathrm{NCO}$ 基团, 受环已烷和 $\alpha$-取 代甲基的位阻作用, 连在环己烷上的仲 NCO 基团的反 应活性比伯 NCO 高 2 3 倍 ${ }^{[21]}$. 为了研究 IPDI 与 HTPB 间的反应, 判断 IPDI 与 HTPB 反应终点, 确保 IPDI 的 仲位 $\mathrm{NCO}$ 基团都与 $\mathrm{HTPB}$ 先反应，而留有伯位 $\mathrm{NCO}$ 基 团待与纳米 $\mathrm{Fe}_{2} \mathrm{O}_{3}$ 表面羟基进行反应，本文采用 $\mathrm{NCO}$ 浓

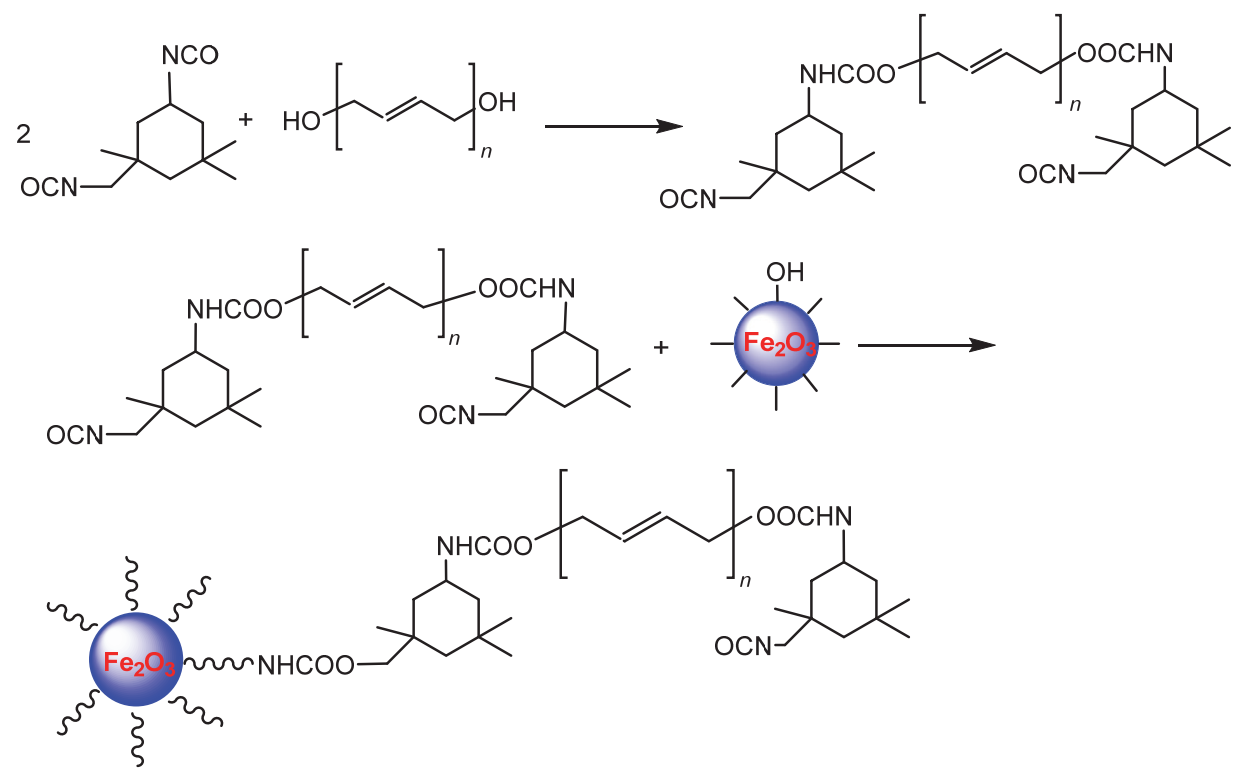

图 1 纳米 $\alpha-\mathrm{Fe}_{2} \mathrm{O}_{3}$ /(IPDI-HTPB)复合粒子合成示意图

Figure 1 The synthesis schematic of $\alpha-\mathrm{Fe}_{2} \mathrm{O}_{3} /(\mathrm{IPDI}-\mathrm{HTPB})$ composite nanoparticles 
度分析法对 HTPB 与 IPDI 的反应动力学进行研究, 其中 $\mathrm{NCO}$ 浓度的测定采用二正丁胺法. 在本实验中, 将 HTPB 与 IPDI 的物质的量比控制为 $1: 1$, 在 60 和 $80{ }^{\circ} \mathrm{C}$ 条件下, 分别对不同时间点反应物中 NCO 浓度进行测 试, 以完成反应级数和反应活化能的测定.

大量研究表明 $\mathrm{OH}$ 与 $\mathrm{NCO}$ 间的反应为二级反应. 当 两种反应物的浓度相等时, 其速率方程的积分形式如公 式 1 所示:

$$
\frac{1}{C_{t}}=k t+\frac{1}{C_{0}}
$$

其中: $k$ 为反应速率常数; $C_{0}$ 为反应物初始浓度; $C_{t}$ 为反 应物在 $t$ 时刻的浓度; $t$ 为反应时间. 以 $C_{t}$ 的倒数对时间 $t$ 作图, 则直线的斜率为反应速率常数, 直线的截距为 初始反应物浓度 $C_{0}$ 的倒数.

图 2 为不同温度下反应物 NCO 浓度与时间的关系 图. 从图中可以看出, 随着反应时间的延长, $\mathrm{NCO}$ 的浓 度降低, 但其降幅逐渐变小, 这主要是因为反应物浓度 的降低导致反应速度降低所致. 对比图 2(a)和图 2(b)发 现, 在相同的反应时间内, $80{ }^{\circ} \mathrm{C}$ 下的-NCO 浓度降幅更 大, 反应速度更快, 因而温度对羟基和异氰酸根的反应 速率影响很大.

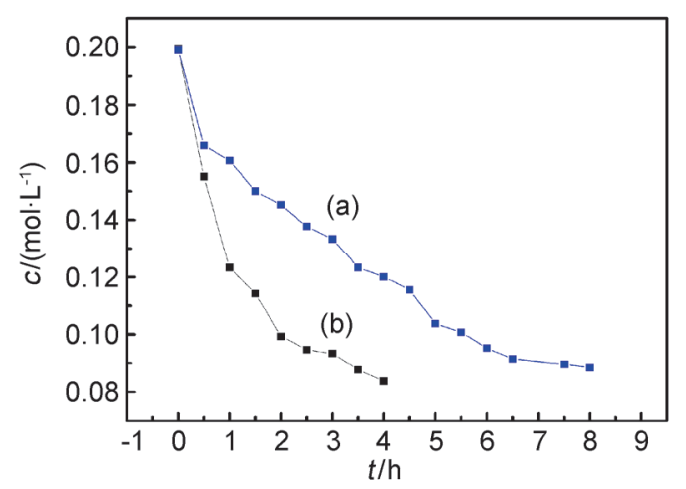

图 2 (a) $60{ }^{\circ} \mathrm{C}$ 和(b) $80{ }^{\circ} \mathrm{C}$ 下应物 $\mathrm{NCO}$ 浓度与时间的关系图 Figure 2 Relation between -NCO concentration and reaction time at (a) $60{ }^{\circ} \mathrm{C}$ and (b) $80{ }^{\circ} \mathrm{C}$

图 3 为 60 和 $80{ }^{\circ} \mathrm{C}$ 下 $\mathrm{NCO}$ 浓度的倒数与反应时间 的关系图. 由图 3 可知, 在 60 和 $80{ }^{\circ} \mathrm{C}$ 下, $\mathrm{NCO}$ 浓度的 倒数与反应时间成直线关系, 这也意味着 $\mathrm{OH}$ 与 $\mathrm{NCO}$ 间的反应为二级反应，这与文献 ${ }^{[22,23]}$ 报道一致. 此外, 在反应的后期, 两者间的直线关系有一定的转折, 这主 要是因为在后一阶段的反应为伯位的 $\mathrm{NCO}$ 与 $\mathrm{OH}$ 反应, 而伯位的 $\mathrm{NCO}$ 其活性低于仲位 NCO, 因而其反应速率 降低, 表现为浓度倒数与时间关系图上的斜率变小. 因 此, 由直线的转折便可以判定活性较强的仲位 NCO 基 团与 $\mathrm{OH}$ 反应的终点, 由图 3 可以看出在 60 和 $80{ }^{\circ} \mathrm{C}$ 条 件下, 仲位 $\mathrm{NCO}$ 与 $\mathrm{OH}$ 反应结束所需时间分别为 6.5 和 $2 \mathrm{~h}$.

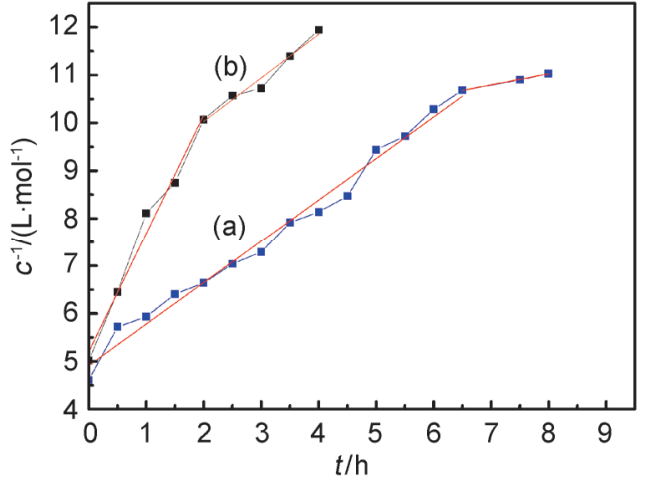

图 3 (a) $60{ }^{\circ} \mathrm{C}$ 和(b) $80{ }^{\circ} \mathrm{C}$ 下反应物 $-\mathrm{NCO}$ 浓度的倒数与时间的关系 图

Figure 3 Relation between the reciprocal of $-\mathrm{NCO}$ concentration and reaction time at (a) $60{ }^{\circ} \mathrm{C}$ and (b) $80{ }^{\circ} \mathrm{C}$

根据公式 1 可以计算得到反应速率常数 $k$, 其中 60 和 $80{ }^{\circ} \mathrm{C}$ 条件下的反应速率常数分别见下表 1.

表 1 IPDI 与 HTPB 二级反应速率常数

Table 1 Second-order rate constants of the reaction between HTPB and IPDI

\begin{tabular}{lll}
\hline 温度 $/{ }^{\circ} \mathrm{C}$ & $k_{1} /\left(\mathrm{L} \cdot \mathrm{mol}^{-1} \cdot \mathrm{h}^{-1}\right)$ & $k_{2} /\left(\mathrm{L} \cdot \mathrm{mol}^{-1} \cdot \mathrm{h}^{-1}\right)$ \\
\hline 60 & 0.7154 & 0.1900 \\
80 & 2.4839 & 0.9116 \\
\hline
\end{tabular}

根据阿伦利乌斯方程的对数形式(见公式 2), 以 $\ln k$ 对 $1 / T$ 作图, 由直线的斜率和截距便可求得该反应的活 化能和指前因子分别为 $E_{\mathrm{a}}=60.82 \mathrm{~kJ} / \mathrm{mol}, A=3.48 \times 10^{9}$.

$$
\ln k=\ln A-\left(E_{\mathrm{a}} / R T\right)
$$

\section{2 纳米 $\alpha-\mathrm{Fe}_{2} \mathrm{O}_{3} /($ IPDI-HTPB)复合粒子的表征}

\subsubsection{XRD 表征}

采用 XRD 分别测定了纯纳米 $\alpha-\mathrm{Fe}_{2} \mathrm{O}_{3}$ 粒子和纳米 $\alpha-\mathrm{Fe}_{2} \mathrm{O}_{3} /($ IPDI-HTPB)复合粒子的晶型, 结果分别如图 4 所示. 对照 JCPDS 标准卡片(No.72-00469), 二组样品在 $24.1^{\circ} 、 33.3^{\circ} 、 35.7^{\circ} 、 40.9^{\circ} 、 49.5^{\circ}$ 和 $54.2^{\circ}$ 等六处都出现 了明显的特征峰, 分别对应于纳米 $\alpha-\mathrm{Fe}_{2} \mathrm{O}_{3}$ 的(012)、 (104)、(101)、(113)、(024)和(116)晶面. 以上结果表明 二组样品为纯度很高的纳米 $\alpha-\mathrm{Fe}_{2} \mathrm{O}_{3}$, 并且经过 HTPB 的包覆后, 复合粒子的晶型与包覆前纯纳米 $\alpha-\mathrm{Fe}_{2} \mathrm{O}_{3}$ 一 样, 复合粒子的晶型并未改变, 但与图 4(a)相比, 图 4(b) 的衍射峰强度明显降低, 这是由于复合粒子的 HTPB 包 覆层所致.

\subsubsection{TEM 分析}

采用 TEM 分别测定了纯纳米 $\alpha-\mathrm{Fe}_{2} \mathrm{O}_{3}$ 和纳米 $\alpha$ $\mathrm{Fe}_{2} \mathrm{O}_{3} /(\mathrm{IPDI}-\mathrm{HTPB})$ 复合粒子的形貌, 结果如图 5 所示. 由图 5(b)和图 5(c) 可知, 在纳米 $\alpha-\mathrm{Fe}_{2} \mathrm{O}_{3} /(\mathrm{IPDI}-\mathrm{HTPB})$ 复 合粒子中, 在纳米 $\alpha-\mathrm{Fe}_{2} \mathrm{O}_{3}$ 的表面有一层 HTPB 包覆层, 


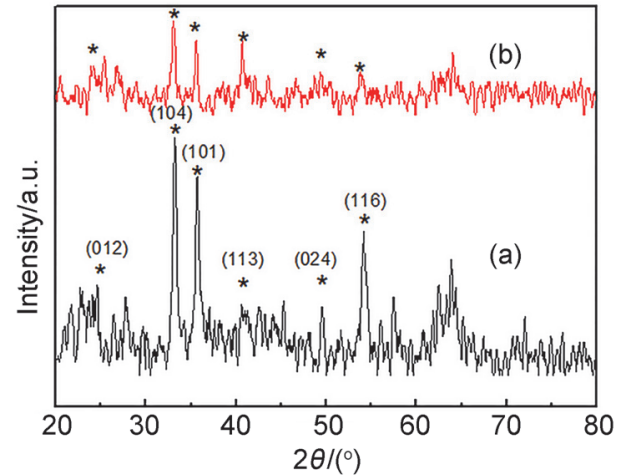

图 4 (a)纯纳米 $\alpha-\mathrm{Fe}_{2} \mathrm{O}_{3}$ 粒子和(b)纳米 $\alpha-\mathrm{Fe}_{2} \mathrm{O}_{3} /(\mathrm{IPDI}-\mathrm{HTPB}$ )复合粒子 的 XRD 图

Figure 4 XRD patterns of (a) pure $\alpha-\mathrm{Fe}_{2} \mathrm{O}_{3}$ nanoparticles and (b) $\alpha$ $\mathrm{Fe}_{2} \mathrm{O}_{3}$ /(IPDI-HTPB) composite nanoparticles
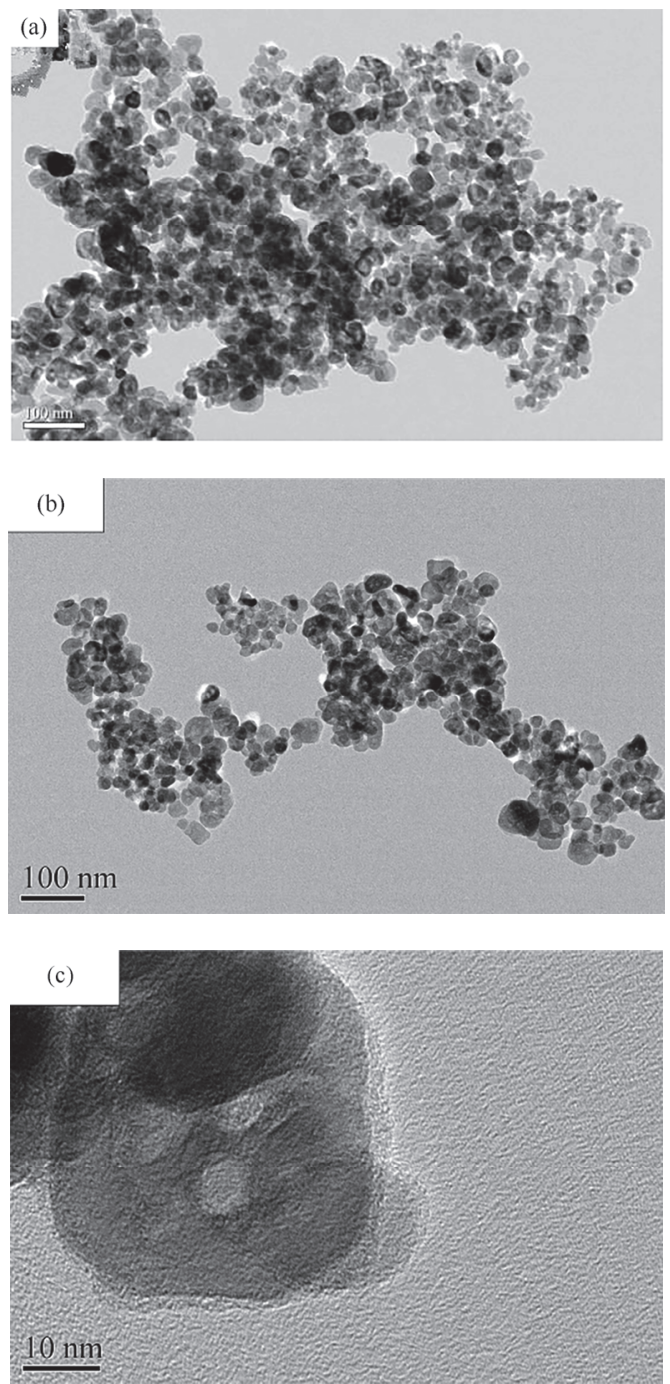

图 5 (a)纯纳米 $\alpha-\mathrm{Fe}_{2} \mathrm{O}_{3}$ 粒子, (b)纳米 $\alpha-\mathrm{Fe}_{2} \mathrm{O}_{3} /($ IPDI-HTPB)复合粒子 和(c)纳米 $\alpha-\mathrm{Fe}_{2} \mathrm{O}_{3} /(\mathrm{IPDI}-\mathrm{HTPB})$ 复合粒子局部放大的 TEM 图

Figure 5 TEM images of (a) pure $\alpha-\mathrm{Fe}_{2} \mathrm{O}_{3}$ nanoparticles, (b) $\alpha-\mathrm{Fe}_{2} \mathrm{O}_{3} /$ (IPDI-HTPB) composite nanoparticles and (c) enlarged detail of $\alpha-\mathrm{Fe}_{2} \mathrm{O}_{3} /$ (IPDI-HTPB) composite nanoparticles
厚度约为 $5 \mathrm{~nm}$. 对比图 5(a)和图 5(b)可看出, 改性后的 复合粒子其分散性得到进一步的提高，这主要是由于 HTPB 的空间位阻作用所致. 由上可知，此种方法能够 将 HTPB 成功接枝在纳米 $\alpha-\mathrm{Fe}_{2} \mathrm{O}_{3}$ 表面, 且包覆层厚度 均匀, 纳米 $\alpha-\mathrm{Fe}_{2} \mathrm{O}_{3}$ 粒子的分散性得到提高.

\subsubsection{FTIR 分析}

为了分析纳米 $\alpha-\mathrm{Fe}_{2} \mathrm{O}_{3}$ 与 IPDI 及 HTPB 间的接枝情 况, 采用 FTIR 分别测定了纳米 $\alpha-\mathrm{Fe}_{2} \mathrm{O}_{3} 、$ IPDI-HTPB 大 分子和纳米 $\alpha-\mathrm{Fe}_{2} \mathrm{O}_{3} /($ IPDI-HTPB)复合粒子, 结果分别 如图 6(a)、图 6(b)和图 6(c)所示. 三组样品在 3428 和 $1639 \mathrm{~cm}^{-1}$ 处均出现明显的吸收峰, 分别对应于样品表 面 $\mathrm{OH}$ 的伸缩振动和弯曲振动. 图 6(a)和图 6(c)在 540 和 $464 \mathrm{~cm}^{-1}$ 处的吸收峰则分别对应于 $\mathrm{Fe}-\mathrm{O}$ 键的伸缩 振动和弯曲振动, 这表明二组样品中均有纳米 $\alpha-\mathrm{Fe}_{2} \mathrm{O}_{3}$ 存在. 由图 6(b) 可以看出, 910、965 和 $993 、 3011 \mathrm{~cm}^{-1}$ 处的特征峰对应于 $\mathrm{RCH}=\mathrm{CH}_{2}$ 的伸缩振动和弯曲振动, $2251 \mathrm{~cm}^{-1}$ 处的特征峰对应 $\mathrm{NCO}$ 的反对称伸缩振动, $1556 \mathrm{~cm}^{-1}$ 处的特征峰对应于氨基甲酸甲酯的 $\mathrm{NH}$ 吸收 峰, 这些峰的出现都表明 HTPB 与 IPDI 间成功反应生成 氨基甲酸甲酯，同时还有 $\mathrm{NCO}$ 基团未参与反应.

与图 6(a)和图 6(b)相比, 图 6(c)中出现了 IPDIHTPB 大分子的峰型, 同时 $\mathrm{NCO} 、 \mathrm{OH}$ 的峰型降低, 表 明其含量变小, 这主要是由于大分子中伯位的 $\mathrm{NCO}$ 与 纳米 $\alpha-\mathrm{Fe}_{2} \mathrm{O}_{3}$ 表面 $\mathrm{OH}$ 接枝反应所致, 这些 IPDI-HTPB 大分子峰型的出现和 NCO 含量的减少都可证实 IPDIHTPB 成功接枝在纳米 $\alpha-\mathrm{Fe}_{2} \mathrm{O}_{3}$ 表面.

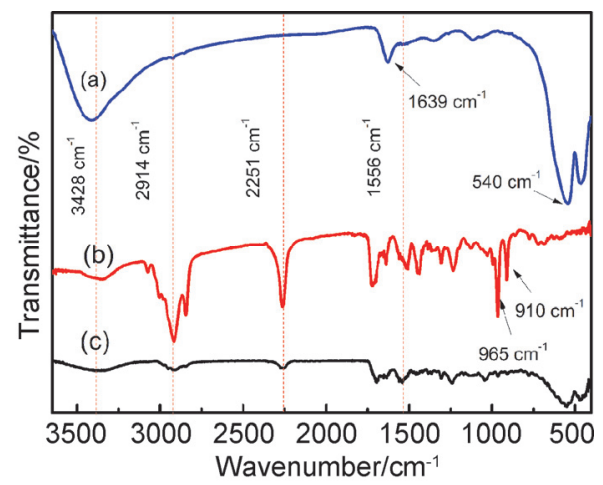

图 6 (a)纳米 $\alpha-\mathrm{Fe}_{2} \mathrm{O}_{3}$ 粒子, (b) IPDI-HTPB 和(c)纳米 $\alpha-\mathrm{Fe}_{2} \mathrm{O}_{3} /(\mathrm{IPDI}-$ HTPB) 复合粒子的 FTIR 图

Figure 6 FTIR spectra of (a) pure $\alpha-\mathrm{Fe}_{2} \mathrm{O}_{3}$ nanoparticles, (b) IPDIHTPB and (c) $\alpha-\mathrm{Fe}_{2} \mathrm{O}_{3} /($ IPDI-HTPB) composite nanoparticles

\subsubsection{TGA 分析}

采用 TGA 测量纳米 $\alpha-\mathrm{Fe}_{2} \mathrm{O}_{3} /($ IPDI-HTPB)复合粒子 中 IPDI-HTPB 含量, 结果如图 7 所示. 由图 7 可见, 复 合粒子的失重过程均分为 3 个阶段, 即: 室温至 $250{ }^{\circ} \mathrm{C}$ 的第一阶段, 对应为复合粒子中纳米 $\alpha-\mathrm{Fe}_{2} \mathrm{O}_{3}$ 表面吸附 水的脱除过程; $250{ }^{\circ} \mathrm{C}$ 至 $380{ }^{\circ} \mathrm{C}$ 的第二阶段, 对应为复 合粒子表面包覆有机层的燃烧过程; 温度 $380{ }^{\circ} \mathrm{C}$ 以上 的第三阶段, 对应为样品的热稳定阶段, 此时复合粒子 
表面有机物已完全燃烧, 失重量不再变化. 由图 7 可知, 纯纳米 $\alpha-\mathrm{Fe}_{2} \mathrm{O}_{3}$ 和纳米 $\alpha-\mathrm{Fe}_{2} \mathrm{O}_{3}$ /(IPDI-HTPB) 复合粒子的 热失重率分别为 $2 \%$ 和 $12 \%$, 这直接表明 HTPB 成功接 枝在纳米 $\alpha-\mathrm{Fe}_{2} \mathrm{O}_{3}$ 的表面，含量约为 $10 \%$.

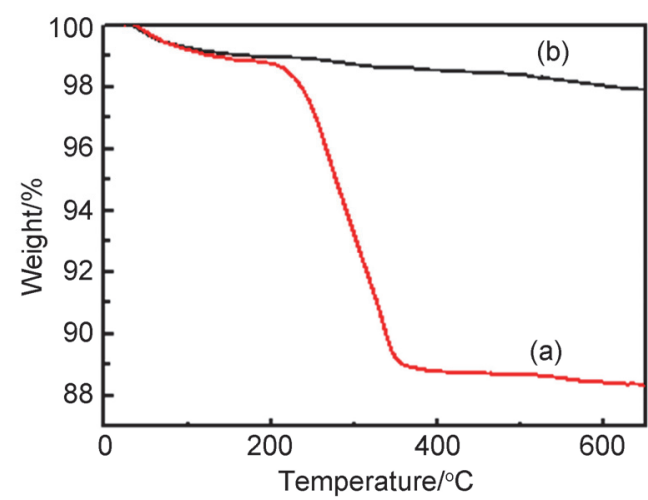

图 7 (a)纳米 $\alpha-\mathrm{Fe}_{2} \mathrm{O}_{3} / \mathrm{HTPB}$ 复合粒子和(b)纯纳米 $\alpha-\mathrm{Fe}_{2} \mathrm{O}_{3}$ 粒子的 TGA 图

Figure 7 TGA images of (a) $\alpha-\mathrm{Fe}_{2} \mathrm{O}_{3} /($ IPDI-HTPB) composite nanoparticles and (b) pure $\alpha-\mathrm{Fe}_{2} \mathrm{O}_{3}$ nanoparticles

\section{2 .5 分散性评估}

为评估纳米 $\alpha-\mathrm{Fe}_{2} \mathrm{O}_{3}$ 在包覆前后的状态变化, 分别 测试了纳米 $\alpha-\mathrm{Fe}_{2} \mathrm{O}_{3} /($ IPDI-HTPB) 复合粒子和纳米 $\alpha-\mathrm{Fe}_{2} \mathrm{O}_{3}$ 粒子在甲苯和水中的分散性, 结果如图 8 所示. 其中图 8(a)和图 8(b)分别为纳米 $\alpha-\mathrm{Fe}_{2} \mathrm{O}_{3} /(\mathrm{IPDI}-\mathrm{HTPB})$ 复合粒子在甲苯和水中静置 $24 \mathrm{~h}$ 后的照片, 图 8(c) 和图 $8(\mathrm{~d})$ 分别为纳米 $\alpha-\mathrm{Fe}_{2} \mathrm{O}_{3}$ 粒子在甲苯和水中静置 $24 \mathrm{~h}$ 后 的照片.

由图 8(a)与 $8(\mathrm{~b})$ 可见, 纳米 $\alpha-\mathrm{Fe}_{2} \mathrm{O}_{3} /($ IPDI-HTPB)复 合粒子可均匀分散于甲苯中表现出亲油性, 却全部漂浮 于水面上表现出疏水性; 而图 8(c)与图 8(d)的结果则表 明, 纳米 $\alpha-\mathrm{Fe}_{2} \mathrm{O}_{3}$ 粒子表现出亲水疏油性. 由此可知, 纳 米 $\alpha-\mathrm{Fe}_{2} \mathrm{O}_{3}$ 经过与 HTPB 的复合后, 材料由亲水疏油性 转变为疏水亲油性.

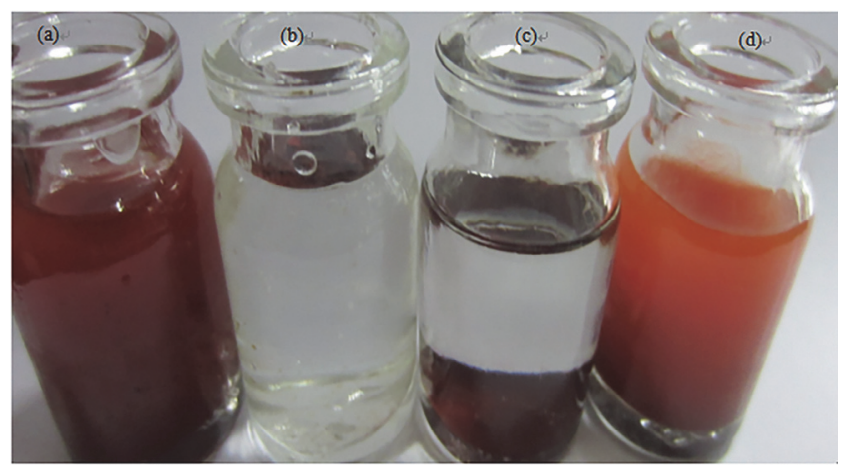

图 8 纳米 $\alpha-\mathrm{Fe}_{2} \mathrm{O}_{3} /\left(\mathrm{IPDI}-\mathrm{HTPB}\right.$ )复合粒子和纯纳米 $\alpha-\mathrm{Fe}_{2} \mathrm{O}_{3}$ 粒子在甲 苯和水中的分散照片

Figure 8 Dispersion of $\alpha-\mathrm{Fe}_{2} \mathrm{O}_{3}$ /(IPDI-HTPB) composite nanoparticles and pure $\alpha-\mathrm{Fe}_{2} \mathrm{O}_{3}$ nanoparticles in toluene and water

(a) $\alpha-\mathrm{Fe}_{2} \mathrm{O}_{3} /\left(\mathrm{IPDI}-\mathrm{HTPB}\right.$ ) composite nanoparticles in toluene; (b) $\alpha-\mathrm{Fe}_{2} \mathrm{O}_{3} /$ (IPDI-HTPB) composite nanoparticles in $\mathrm{H}_{2} \mathrm{O}$; (c) pure $\alpha-\mathrm{Fe}_{2} \mathrm{O}_{3}$ nanoparticles in toluene; (d) pure $\alpha-\mathrm{Fe}_{2} \mathrm{O}_{3}$ nanoparticles in $\mathrm{H}_{2} \mathrm{O}$
依据相似相容原理, 在以 HTPB 为粘结剂的推进剂 产品中, 纳米 $\alpha-\mathrm{Fe}_{2} \mathrm{O}_{3} / \mathrm{HTPB}$ 复合粒子由于表面 HTPB 的存在而更易分散到推进剂中，从而实现纳米 $\alpha-\mathrm{Fe}_{2} \mathrm{O}_{3}$ 粒子在推进剂中的均匀分散.

\subsection{6 催化性能测定}

采用纳米 $\alpha-\mathrm{Fe}_{2} \mathrm{O}_{3}$ 改性前后对 $\mathrm{AP}$ 的热分解的影响 来验证其催化性能. 制备了三个样品, 分别为纯 AP 样 品(标记为样品一)、AP 中加入纳米 $\alpha-\mathrm{Fe}_{2} \mathrm{O}_{3} /(\mathrm{IPDI}-\mathrm{HTPB})$ 复合粒子样品(标记为样品二)和 $\mathrm{AP}$ 与纯纳米 $\alpha-\mathrm{Fe}_{2} \mathrm{O}_{3}$ 、 HTPB 的简单混合样品(标记为样品三). 其中, 样品三 中 $\alpha-\mathrm{Fe}_{2} \mathrm{O}_{3} 、 \mathrm{HTPB}$ 以及 AP 的量与样品二中都相同, 样 品二和样品三中纳米 $\alpha-\mathrm{Fe}_{2} \mathrm{O}_{3}$ 的组分与 $\mathrm{AP}$ 质量比均为 $2 \%$. 以上三个样品的热分解结果分别如图 9(a)、(b)、(c) 所示. 由图 9(a)可知: 纯 AP 的低温放热峰和高温放热峰 分别为 315 和 $460{ }^{\circ} \mathrm{C}$. 由图 9(b)和图 9(c)可知, 样品二 和样品三中, AP 的低温分解峰都消失, 高温分解峰温都 明显降低. 样品二中纳米 $\alpha-\mathrm{Fe}_{2} \mathrm{O}_{3} /($ IPDI-HTPB)复合粒 子可使 AP 热分解的高温分解峰温降低 $109{ }^{\circ} \mathrm{C}$, 样品三 中纯纳米 $\mathrm{Fe}_{2} \mathrm{O}_{3}$ 可使 $\mathrm{AP}$ 热分解的高温分解峰温降低 $99{ }^{\circ} \mathrm{C}$. 对比样品二和样品三的热分解结果可知: 与纯 纳米 $\alpha-\mathrm{Fe}_{2} \mathrm{O}_{3}$ 相比, 纳米 $\alpha-\mathrm{Fe}_{2} \mathrm{O}_{3} /($ IPDI-HTPB)复合粒子 可使 $\mathrm{AP}$ 的高温分解峰温进一步降低 $10{ }^{\circ} \mathrm{C}$, 这表明纳 米 $\alpha-\mathrm{Fe}_{2} \mathrm{O}_{3} /(\mathrm{IPDI}-\mathrm{HTPB})$ 复合粒子对 AP 的热分解性能优 于纯纳米 $\alpha-\mathrm{Fe}_{2} \mathrm{O}_{3}$.

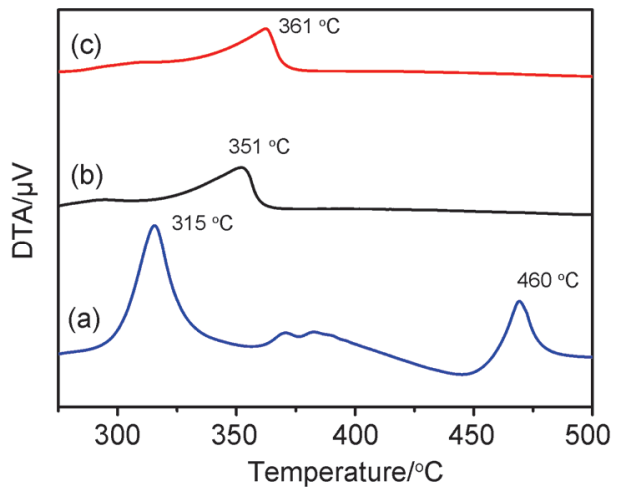

图 9 不同样品混合 $\mathrm{AP}$ 后的 DTA 图. (a)纯 AP; (b) $\mathrm{AP}+$ 纳米 $\alpha-\mathrm{Fe}_{2} \mathrm{O}_{3}$ / (IPDI-HTPB)复合粒子; (c)纳米 $\alpha-\mathrm{Fe}_{2} \mathrm{O}_{3} 、 \mathrm{HTPB}$ 和 AP 的简单混合样 Figure 9 DTA images of different samples. (a) pure AP; (b) AP+ $\alpha-\mathrm{Fe}_{2} \mathrm{O}_{3} /($ IPDI-HTPB) composite nanoparticles; (c) simply mixing pure $\alpha-\mathrm{Fe}_{2} \mathrm{O}_{3}$ nanoparticles, HTPB and AP

$\alpha-\mathrm{Fe}_{2} \mathrm{O}_{3}$ 属于过渡金属氧化物，是 $n$-型半导体，它的 半导体性质与它的催化活性都有着密切关系, $\alpha-\mathrm{Fe}_{2} \mathrm{O}_{3}$ 对 AP 催化作用主要是通过氧化还原循环中加速电子转 移，使 AP 提前分解，从而促进 AP 系固体推进剂燃速的 提高 ${ }^{[24]}$. 在 AP 的热分解中以下三个过程起着决定性作 用 ${ }^{[25]}$ :
(1) 电子由 $\mathrm{ClO}_{4}{ }^{-}$转给 $\mathrm{NH}_{4}{ }^{+}$

$\mathrm{NH}_{4}{ }^{+}+\mathrm{ClO}_{4}{ }^{-} \rightarrow \mathrm{NH}_{4}+\mathrm{ClO}_{4}$ 
(2) 质子由 $\mathrm{NH}_{4}{ }^{+}$转给 $\mathrm{ClO}_{4}$

$\mathrm{NH}_{4}{ }^{+}+\mathrm{ClO}_{4}{ }^{-} \rightarrow \mathrm{NH}_{3}+\mathrm{HClO}_{4}$

(3) 由于 $\mathrm{Cl}-\mathrm{O}$ 键的断裂而导致的 $\mathrm{ClO}_{4}{ }^{-}$热分解

$\mathrm{C} 1 O_{4}{ }^{-} \rightarrow \mathrm{ClO}_{3}{ }^{-}+\mathrm{O}$

Winfried 等 ${ }^{[26]}$ 认为过渡金属氧化物对 AP 的催化作 用可能是由于氧化物的多价态性而导致氧化还原过程 中的电荷迁移速度加快而引起的. 在过渡金属氧化的催 化过程中，有金属高氯化物中间体的形成，这些中间体 在热力学上是不稳定的, 它们会降低 AP 热分解的初始 活化能, 从而加速 AP 的热分解. 本课题组 ${ }^{[27]}$ 的前期工 作也证明了这一点.

在本研究中, 样品二和样品三中, 都存在 $\alpha-\mathrm{Fe}_{2} \mathrm{O}_{3}$ 纳米粒子; 样品三中, $\alpha-\mathrm{Fe}_{2} \mathrm{O}_{3} 、 \mathrm{HTPB}$ 和 AP 是简单混合, $\alpha-\mathrm{Fe}_{2} \mathrm{O}_{3}$ 粒子会团聚、分散性较差, 导致纳米 $\alpha-\mathrm{Fe}_{2} \mathrm{O}_{3}$ 对 AP 热分解的催化性能降低. 而样品二中, 纳米 $\alpha-\mathrm{Fe}_{2} \mathrm{O}_{3}$ 经过 IPDI-HTPB 改性后, 纳米粒子间不会发生团聚, 可 提高纳米 $\alpha-\mathrm{Fe}_{2} \mathrm{O}_{3}$ 对 $\mathrm{AP}$ 热分解的催化性能.

\section{3 结论}

HTPB 与 IPDI 间的动力学研究显示, 两者间的反应 为典型的二级反应, 其反应的活化能为 $E_{\mathrm{a}}=60.82 \mathrm{~kJ} /$ $\mathrm{mol}$, 指前因子为 $A=3.48 \times 10^{9}$.

通过 IPDI 偶联, 可将 HTPB 成功地接枝在纳米 $\alpha-\mathrm{Fe}_{2} \mathrm{O}_{3}$ 表面, 包覆层厚度约为 $5 \mathrm{~nm}$; 纳米 $\alpha-\mathrm{Fe}_{2} \mathrm{O}_{3}$ 经过 与 HTPB 的复合后, 由亲水疏油性转变为疏水亲油性; 改性后的纳米 $\alpha-\mathrm{Fe}_{2} \mathrm{O}_{3}$ 复合粒子的分散性得到大幅提高. 与纯纳米 $\alpha-\mathrm{Fe}_{2} \mathrm{O}_{3}$ 相比, 纳米 $\alpha-\mathrm{Fe}_{2} \mathrm{O}_{3} /(\mathrm{IPDI}-\mathrm{HTPB}$ )复合 粒子对 AP 具有更好的热分解催化作用.

\section{4 实验部分}

\section{1 实验原料}

HTPB，黎明化工研究院; $\alpha-\mathrm{Fe}_{2} \mathrm{O}_{3}$, 北京纳辰科技 有限公司, 纯度 $99.9 \%$, 粒径 $30 \mathrm{~nm}$; IPDI，上海晶纯实 业有限公司, AR 级; 异丙醇, 国药集团化学试剂有限公 司, AR 级; 二正丁胺, 江苏永华精细化学品有限公司, $\mathrm{CP}$ 级; 溴酚蓝, 天津市化学试剂研究院, AR 级; 无水乙 醚, 国药集团化学试剂有限公司, AR 级; 甲苯, 国药集 团化学试剂有限公司, AR 级.

\section{2 实验方法}

\subsection{1 溶液中 NCO 含量的测定}

溶液中 NCO 含量采用二正丁胺法进行测定. 取一 个干燥洁净的 $250 \mathrm{~mL}$ 雉形瓶, 用移液管向其中加入 25 $\mathrm{mL}$ 二正丁胺无水甲苯溶液 $(0.1 \mathrm{~mol} / \mathrm{L})$, 另取一支移液 管准确量取 $10 \mathrm{~mL}$ 待测样品加入雉形瓶中, 摇晃 15 $\mathrm{min}$, 然后加入 $100 \mathrm{~mL}$ 异丙醇, 向雉形瓶中滴加 $4 \sim 6$ 滴溴酚蓝指示剂 $(1 \mathrm{~g} / \mathrm{mL})$ 并摇勺, 最后用 $0.1 \mathrm{~mol} / \mathrm{L}$ 盐酸 标准溶液进行滴定, 待溶液颜色由蓝色转变为黄色, 并
保持黄色 $15 \mathrm{~s}$ 不变时, 确认此时为滴定终点并记录盐酸 的用量. 采用同样的方法做无待测样品的空白实验，并 记录空白实验盐酸用量.

\subsection{2 纳米 $\alpha-\mathrm{Fe}_{2} \mathrm{O}_{3} /(\mathrm{IPDI}-\mathrm{HTPB})$ 复合粒子的制备}

取两个干燥洁净的烧杯，分别称取 $2.22 \mathrm{~g}$ IPDI (10 $\mathrm{mmol}$ )和 $16.11 \mathrm{~g} \mathrm{HTPB}(10 \mathrm{mmol})$, 向两个烧杯中分别 加入 $20 \mathrm{~mL}$ 和 $70 \mathrm{~mL}$ 甲苯, 在玻璃棒机械搅拌作用下将 HTPB 完全溶解于甲苯溶液, 然后将两个烧杯中的溶液 加入 $150 \mathrm{~mL}$ 圆底烧瓶中, 搅拌作用下滴加辛酸亚锡催 化剂, 在 $\mathrm{N}_{2}$ 保护下缓慢升高温度至 $80{ }^{\circ} \mathrm{C}$, 反应 $2 \mathrm{~h}$, 待 用.

再取一个干燥洁净的烧杯, 准确称取 $0.10 \mathrm{~g}$ 烘干的 纳米 $\alpha-\mathrm{Fe}_{2} \mathrm{O}_{3}$, 量取 $30 \mathrm{~mL}$ 甲苯加入烧杯中, $50 \mathrm{kHz}$ 频率 下超声 $10 \mathrm{~min}$, 待纳米 $\alpha-\mathrm{Fe}_{2} \mathrm{O}_{3}$ 在甲苯溶液中分散后, 将溶液加入上一步骤的圆底烧瓶中，反应 $4 \mathrm{~h}$. 待反应 结束后, 将溶液与复合粒子离心分离, 分离出的固相用 甲苯和乙醚多次清洗, 产物在 $80{ }^{\circ} \mathrm{C}$ 烘箱中干燥 $12 \mathrm{~h}$, 最终制备得到纳米 $\alpha-\mathrm{Fe}_{2} \mathrm{O}_{3} /(\mathrm{IPDI}-\mathrm{HTPB})$ 复合粒子.

\section{3 表征手段}

复合粒子的形貌与分散状况采用 JEM2100CX (Jeol 日本)透射电子显微镜(TEM)表征，分散介质为乙醇; 复 合粒子有机包覆量在 TGA209F3 型(Netzsch 德国)热重 分析仪(TGA)上进行测定, 升温速度为 $10{ }^{\circ} \mathrm{C} / \mathrm{min}$, 升温 范围为室温 $\sim 700{ }^{\circ} \mathrm{C}$, 铝质池; 复合粒子表面有机层采 用 AVATAR 360 (Nicolet 美国)傅里叶变换红外光谱仪 (FTIR)分析, $\mathrm{KBr}$ 压片，测量范围 $400 \sim 4000 \mathrm{~cm}^{-1}$; 复合 粒子晶型结构采用 Bruker D8 型(Bruker 德国)X 射线粉 末衍射(XRD)分析, $\mathrm{Cu}$ 靶 $\mathrm{K} \alpha$ 射线，扫描范围 $20^{\circ} \sim 80^{\circ}$, 扫描速度 $3\left(^{\circ}\right) / \mathrm{min}$, 步长 $0.02^{\circ}$.

\section{References}

[1] Wu, J. J.; Ji, Z. Y.; Shen, X. P.; Miao, X. L.; Xu, K. Q. Acta Chim. Sinica 2017, 75, 1207 (in Chinese). (吴佳佳, 季振源, 沈小平, 缪 绪立, 徐克强, 化学学报, 2017, 75, 1207.)

[2] Mu, Y.; Jia, F. L.; Ai, Z. H.; Zhang, L. Z. Acta Chim. Sinica 2017 75,538 (in Chinese). (穆毅, 贾法龙, 艾智慧, 张礼知, 化学学报, 2017, 75, 538.)

[3] Cui, S. Z.; Yang, H. P.; Sun, H. H.; Nie, K.; Wu, J. M. Acta Chim. Sinica 2016, 74, 995 (in Chinese). (崔素珍, 杨汉培, 孙慧华, 聂 坤, 吴俊明, 化学学报, 2016, 74, 995.)

[4] Padwal, M. B.; Varmal, M. Combust. Sci. Technol. 2018, 190, 1614.

[5] Chang, H. Z.; Zhang, T.; Dang, H.; Chen, X. Y.; You, Y. C.; Schwank, J.; Li, J. H. Catal. Sci. Technol. 2018, 8, 3313.

[6] Niu, H. H.; Zhang, S. W.; Ma, Q.; Qin, S. X.; Wan, L.; Xu, J. Z.; Miao, S. D. RSC Adv. 2013, 3, 17228.

[7] Narayani, H.; Jose, M.; Sriram, K.; Shukla, S. Environ. Sci. Pollut. Res. 2018, 25, 20304

[8] Shahrousvand, M.; Hoseinian, M. S.; Ghollasi, M.; Karbalaeimahdi, A.; Salimi, A.; Tabar, F. A. Mater. Sci. Eng. C 2017, 74, 556.

[9] Ke, X.; Zhou, X.; Hao, G. Z.; Xiao, L.; Liu, J.; Jiang, W. Appl. Surf. Sci. 2017, 407, 137

[10] Xu, H.; Wang, X. B.; Zhang, L. Z. Powder Technol. 2008, 185, 176.

[11] Song, L. M.; Zhang, S. J.; Chen, B.; Ge, J. J.; Jia, X. C. Colloids Surf. A 2010, 360, 1 .

[12] Atta, A. H.; El-ghamry, M. A.; Hamzaoui, A.; Refat, M. S. J. Mol Struct. 2015, 1086, 246 .

[13] Jadhav, S. A.; Bongiovanni, R. L.; Marchisio, D.; Fontana, D.; Eg- 
ger, C. Pigm. Resin Technol. 2014, 43, 219.

[14] Iida, H.; Nakanishi, T.; Osaka, T. Electrochim. Acta 2005, 51, 855

[15] Zhan, J. Y.; Tian, G. F.; Jiang, L. Z.; Wu, Z. P.; Wu, D. Z.; Yang, X. P. Thin Solid Films 2008, 516, 6315.

[16] Liu, J.; Ke, X.; Xiao, L.; Hao, G. Z.; Rong, Y. B.; Jin, C. S.; Jiang, W.; Li, F. S. Propellants Explos. Pyrotech. 2018, 43, 144.

[17] Jin, B.; Peng, R.; Zhao, F.; Yi, J.; Xu, S.; Wang, S.; Chu, S. Propellants Explos. Pyrotech. 2014, 39, 874.

[18] Lu, Y. W.; Zhu, Y. F.; Xu, P. F.; Ye, P.; Gao, B.; Sun, Y.; Guo, C. P. J. Solid State Chem. 2018, 258, 718.

[19] Isert, S.; Groven, L. J.; Lucht, R. P.; Son, S. F. Combust. Flame 2015, 162, 1821 .

[20] Hu, L; Ma, Z. Y.; Ji, M. W.; Zhang, L. X. Acta Chim. Sinica 2011, 69, 3028 (in Chinese). (胡否, 马振叶, 纪明卫, 张利雄, 化学学
报, 2011, 69, 3028.)

[21] Yin, L.; Chu, J. L. Paint Coat. Ind. 1999, 29, 34.

[22] Hailu, K.; Guthausen, G.; Becker, W.; König, A.; Bendfeld, A.; Geissler, E. Polym. Test. 2010, 29, 513.

[23] Catherine, K. B.; Krishnan, K.; Nina, K. B. J. Therm. Anal. Calorim. 2000, 59, 93.

[24] Yang, R. J.; Ma, Q. Y. J. Solid Rocket Technol. 1991, 9, 78 (in Chinese). (杨荣杰, 马庆云, 固体火箭技术, 1991, 9, 78.)

[25] Ganga, T.; Devi, G. T.; Kannan, M. P.; Hema, B. Thermochim. Acta 1996, 285, 269

[26] Winfried, K. R.; Eli, S. F. J. Phys. Chem. 1970, 74, 3317.

[27] Ma, Z. Y.; Wu, R. J.; Song, J.; Li, C.; Chen, R. Z.; Zhang, L. X. Propellants Explos. Pyrotech. 2012, 37, 183.

(Cheng, F.) 